PROCEEDINGS OF THE

AMERICAN MATHEMATICAL SOCIETY

Volume 132, Number 5, Pages 1355-1366

S 0002-9939(03)07088-6

Article electronically published on December 12, 2003

\title{
EXISTENCE OF SOLUTIONS FOR SEMILINEAR ELLIPTIC PROBLEMS WITHOUT (PS) CONDITION
}

\author{
JIANFU YANG
}

(Communicated by David S. Tartakoff)

\begin{abstract}
We establish an existence result for semilinear elliptic problems with the associated functional not satisfying the Palais-Smale condition. The nonlinearity of our problem does not satisfy the Ambrosetti-Rabinowitz condition.
\end{abstract}

\section{INTRODUCTION}

Existence of solutions for the semilinear elliptic Dirichlet problem

$$
-\Delta u=f(x, u) \quad \text { in } \Omega, u=0 \quad \text { on } \partial \Omega,
$$

where $\Omega$ is a bounded domain in $\mathbb{R}^{N}, N \geq 2$, has been an object of intensive research in recent years. In general, there are two approaches used in the study of the problem. One is the variational method originated by Ambrosetti and Rabinowitz [1]. Besides geometrical assumptions on the associated functional of (1.1), they require the so-called Palais-Smale condition ((PS) condition for short). In the verification of the Palais-Smale condition, Ambrosetti and Rabinowitz introduced the following condition:

$$
0<F(x, t) \leq \theta t f(x, t)
$$

for $|t|$ large, where $\theta \in\left(0, \frac{1}{2}\right)$. There are some modifications [6] of this condition. However, it is difficult to remove the condition completely in obtaining existence results although it seems a technical condition. Another approach is topological methods. This requires the establishing of a priori bounds for eventual solutions of the problem. In [4] a strong restriction on the growth of $f(x, u)$ at infinity with respect to $u$ was required. Later this restriction was lifted in [7, [10]. However, in both cases, a certain behavior of the nonlinearity $f$ at infinity was necessary. In [10] the nonlinearities had to be essentially a power at infinity, while in [7] some sort of mild oscillation was allowed. A priori bounds are also obtained in [2] and 14] for nonlinearities as powers $|t|^{p-2} t$ at infinity by using the information of the Morse index of the solutions.

In a recent work 8], de Figueiredo and the author proved an existence result for (1.1). It allows that the nonlinearity $f$ oscillates at infinity between powers

Received by the editors May 4, 2002 and, in revised form, September 18, 2002.

2000 Mathematics Subject Classification. Primary 35J20, 35J25, 35J60.

Key words and phrases. Palais-Smale condition, semilinear, elliptic problem.

(C)2003 American Mathematical Society 
$t^{p}$, and then the associated functional may not satisfy the Palais-Smale condition. The arguments used in this work are a combination of variational methods with a use of the estimation of the Morse index in the blow-up method. In this work, $\liminf \operatorname{int}_{t \rightarrow+\infty} \frac{f(x, t)}{t^{p}}>0$ is required. Our aim in this paper is to establish the existence of positive solutions and multiple solutions for problem (1.1). Besides allowing $f$ to oscillate, the limit $\lim \inf _{t \rightarrow+\infty} \frac{f(x, t)}{t^{p}}$ may be zero. In this case, blow-up arguments in [8] will lead to a problem in the whole space which possibly has a solution. Then it fails to work. The type of hypotheses assumed here do not imply a (PS) condition. Also they do not fit in the conditions that imply a priori bounds. Suppose that $f$ satisfies

(f1) $f \in C^{1}(\bar{\Omega} \times \mathbb{R}), f(x, 0)=f_{t}^{\prime}(x, 0)=0$, for all $x \in \bar{\Omega}$.

(f2) There exist $T>0,1<p<\frac{N+2}{N-2}$ if $N \geq 3$ and $p \in(1, \infty)$ if $N=2$ and a positive bounded nonincreasing function $\bar{h}$ such that

$$
\left|f_{t}^{\prime}(x, t)\right| \leq C|t|^{p-1} \text { and }\left|\nabla_{x} f(x, t)\right| \leq C\left(\bar{h}(t)|t|^{p}+1\right)
$$

for $|t| \geq T$ and $x \in \bar{\Omega}$.

We may write $f$ in the form

$$
f(x, t)=h(x, t)|t|^{p-1} t+g(x, t) .
$$

Condition (f2) implies $h(x, t), h_{t}^{\prime}(x, t) t+p h(x, t)$ are bounded and $\lim \sup _{t \rightarrow \infty} \frac{h^{\prime}(x, t) t}{h(x, t)}$ $\leq 0$.

(f3) There exists a bounded nonincreasing function $\underline{h}$ such that

$$
\begin{gathered}
0<\underline{h}(t) \leq h(x, t) \leq \bar{h}(t), \quad 0<\lim _{t \rightarrow \infty} \frac{\underline{h}(t)}{\bar{h}(t)}<+\infty, \\
-p<\liminf _{t \rightarrow \infty} \frac{h_{t}^{\prime}(x, t) t}{h(x, t)} \leq \limsup _{t \rightarrow \infty} \frac{h_{t}^{\prime}(x, t) t}{h(x, t)} \leq 0, \quad-p<\lim _{t \rightarrow \infty} \frac{\bar{h}^{\prime}(t) t}{\bar{h}(t)} \leq 0
\end{gathered}
$$

and $h^{\prime}(x, t) t \rightarrow 0$ as $t \rightarrow \infty$.

(f4) There exist a constant $\nu>0$ and $1<q \leq p$ such that

$$
\nu \leq \liminf _{t \rightarrow+\infty} \frac{g(x, t) t}{|t|^{q+1}} \leq \limsup _{t \rightarrow+\infty} \frac{g(x, t) t}{|t|^{q+1}}<+\infty
$$

uniformly in $x \in \Omega$.

If $h$ is bounded below by a positive constant or $\lim \sup _{t \rightarrow \infty} h(x, t) t^{p-q}$ is finite, the existence of positive solutions is a consequence of the result in [8]. But here we may allow $h(x, t)$ to oscillate and to tend to zero as $t$ goes to infinity. This case is exclusive in previous works.

Our result is concerned with the existence of a positive solution as well as existence of multiple solutions of problem (1.1).

Theorem 1.1. Suppose (f1)-(f4). Problem (1.1) possesses at least a positive solution if $f(x, t) \geq 0$ for $t \geq 0$ uniform in $x$; it possesses infinitely many solutions if $f(x, t)=-f(x,-t)$.

We remark that conditions (f2) and (f3) imply that for some new constant $C>0$,

$$
|f(x, t)| \leq C\left(1+t^{p}\right), \forall t \geq 0 .
$$

So the nonlinearity $f(t)$ is superlinear, but the ratio $f(t) / t^{p}$ is not required to converge at infinity. In general, the existence of a positive solution of (1.1) requires 
either that the domain $\Omega$ be convex, or more generally, that $\Omega$ have some special geometrical properties, or $\frac{f(t)}{t}$ is monotone in $t$ even in the case that $f$ is independent of $x$. We do not need these conditions here. As an example, the function

$$
f(t)=\frac{1}{1+\ln ^{2} t}(\gamma+\sin \ln t) t^{p}+g(x, t), \quad \gamma>1
$$

for $t>0$ with lower growth term $g(x, t)$ as in the example in [8] satisfies our assumptions but they fit neither the Ambrosetti-Rabinowtz condition nor condition (f5) in 7]. In [18] Zou obtained some multiplicity results without the condition (1.2). However, he also assumed that $\frac{f(t)}{t}$ is increasing in $t$.

The method of proof of our results uses the "monotonicity trick" developed by M. Struwe [17] and L. Jeanjean [13]. Firstly, we consider the parametrized problem

$$
-\Delta u=\lambda f(x, u) \quad \text { in } \Omega, u=0 \quad \text { on } \partial \Omega, \lambda \in[1,2] .
$$

Using the "monotonicity trick" we may find a solution of (1.4) for almost every $\lambda \in[1,2]$; next, we choose a sequence $\left\{\lambda_{n}\right\}$ such that $\lambda_{n} \rightarrow 1$ as $n \rightarrow \infty$. If the corresponding solutions $u_{n}:=u_{\lambda_{n}}$ converge in $H_{0}^{1}(\Omega)$, then we are done. The idea for proving the convergence of $\left\{u_{n}\right\}$ is to obtain an $L^{\infty}$-bound of $\left\{u_{n}\right\}$. This involves an estimation of the Morse index $\operatorname{Ind}\left(u_{\lambda}\right)$ of $u_{\lambda}$. It is well known that there exists a solution at the mountain-pass level with the Morse index less than or equal to 1 if the Palais-Smale condition is satisfied. However, it is not clear if the functional associated to (1.4) satisfies the Palais-Smale condition. We observe that we still have the Morse index $\operatorname{Ind}\left(\mathrm{u}_{\lambda}\right) \leq 1$ in our case. The step of proving that $\left\{u_{n}\right\}$ is bounded involves an argument by contradiction, which leads to the question of existence of nontrivial solutions of certain problems in $\mathbb{R}^{N}$, or in half-spaces. Those questions are commonly called Liouville-type theorems. We will state Liouvilletype theorems in section 2. We also give a precise statement of abstract critical point theorems with the estimate of Morse indices of critical points in section 2 . Theorem 1.1 is proved in section 3 .

\section{Preliminary Results}

Let $X$ be a Banach space equipped with the norm $\|\cdot\|$ and $\varphi$ be a $C^{1}$ functional on $X$. Consider a homotopy-stable family $\mathcal{F}$ of compact subsets of $X$ with a closed boundary $B$ (see [9] for the definition). Set

$$
c(\varphi, \mathcal{F}):=\inf _{A \in \mathcal{F}} \max _{x \in A} \varphi(x) .
$$

We say that $\left\{A_{n}\right\} \subset \mathcal{F}$ is min-maxing for $\varphi$ if $\lim _{n} \sup _{A_{n}} \varphi=c(\varphi, \mathcal{F})$, and we say that $\varphi$ satisfies $(P S)_{c}$ along a sequence $\left(A_{n}\right)_{n}$ in $\mathcal{F}$ if every sequence $\left\{x_{n}\right\}$ that verifies $\varphi\left(x_{n}\right) \rightarrow c, \varphi^{\prime}\left(x_{n}\right) \rightarrow 0$ and $\lim _{n} \operatorname{dist}\left(x_{n}, A_{n}\right)=0$ has a convergent subsequence. A sequence $\left\{x_{n}\right\}$ is near $\left\{A_{n}\right\}$ whenever $\liminf _{n} d\left(x_{n}, A_{n}\right) \rightarrow 0$.

Let $\Lambda \in \mathbb{R}^{+}$be an interval. A family of $C^{1}$-functionals $\left(I_{\lambda}\right)_{\lambda \in \Lambda}$ on $X$ is given by

$$
I_{\lambda}(u):=A(u)-\lambda B(u), \quad \forall \lambda \in \Lambda,
$$

where $B(u) \geq 0$, for all $u \in X$ and we assume either $A(u) \rightarrow+\infty$ or $B(u) \rightarrow+\infty$ as $\|u\| \rightarrow \infty$ and

$$
c_{\lambda}:=\inf _{\{\gamma \in \Gamma\}} \max _{\{0 \leq t \leq 1\}} I_{\lambda}(\gamma(t))>\max \left\{I_{\lambda}\left(u_{1}\right), I_{\lambda}\left(u_{2}\right)\right\}
$$

for some $u_{1}, u_{2} \in X$, where $\Gamma=\left\{\gamma \in C([0,1], X), \gamma(0)=u_{1}, \gamma(1)=u_{2}\right\}$. The following result is due to Jeanjean [13]. 
Proposition 2.1. For almost every $\lambda \in \Lambda$, there exists a bounded min-maxing sequence $\left\{A_{n}\right\}$ and a bounded sequence $\left\{v_{n}\right\} \subset X$ near $\left\{A_{n}\right\}$ such that

$$
I_{\lambda}\left(v_{n}\right) \rightarrow c_{\lambda} \text { and } I_{\lambda}^{\prime}\left(v_{n}\right) \rightarrow 0
$$

as $n \rightarrow \infty$.

Next we state the multiplicity results.

Suppose $X=\overline{\bigoplus_{j \in \mathbb{N}} X_{j}}$ with $\operatorname{dim} X_{j}<\infty$ for any $j \in \mathbb{N}$. Set $Y_{k}=\overline{\bigoplus_{j=0}^{k} X_{j}}, Z_{k}=$ $\overline{\bigoplus_{j \in \mathbb{N}}^{\infty} X_{j}}$ and

$$
B_{k}=\left\{u \in Y_{k}:\|u\| \leq \rho_{k}\right\}, N_{k}=\left\{u \in Z_{k}:\|u\|=r_{k}\right\}
$$

for $\rho_{k}>r_{k}>0$. We assume further that $I_{\lambda}$ maps bounded sets to bounded sets uniformly for $\lambda \in[1,2]$ and $I_{\lambda}(-u)=I_{\lambda}(u)$ for all $(\lambda, u) \in[1,2] \times X$. Let, for $k \geq 2$,

$$
c_{k}(\lambda):=\inf _{\gamma \in \Gamma_{k}} \max _{u \in B_{k}} I_{\lambda}(\gamma(u)),
$$

where $\Gamma_{k}=\left\{\gamma \in C\left(B_{k}, X\right): \gamma\right.$ is odd, $\left.\left.\gamma\right|_{\partial B_{k}}=i d\right\}$,

$$
\begin{aligned}
b_{k}(\lambda) & :=\inf _{u \in Z_{k},\|u\|=r_{k}} I_{\lambda}(u), \\
a_{k}(\lambda) & :=\max _{u \in Y_{k},\|u\|=\rho_{k}} I_{\lambda}(u) .
\end{aligned}
$$

Zou [18] proved the following result.

Proposition 2.2. If $b_{k}(\lambda)>a_{k}(\lambda)$ for all $\lambda \in[1,2]$, then $c_{k}(\lambda) \geq b_{k}(\lambda)$ for all $\lambda \in[1,2]$. Moreover, for almost every $\lambda \in[1,2]$, there exists a bounded min-maxing sequence $\left\{A_{n}^{k}\right\}$ and a bounded sequence $\left\{u_{n}^{k}\right\} \subset X$ near $\left\{A_{n}^{k}\right\}$ such that

$$
I_{\lambda}\left(u_{n}^{k}\right) \rightarrow c_{k}(\lambda) \text { and } I_{\lambda}^{\prime}\left(u_{n}^{k}\right) \rightarrow 0
$$

as $n \rightarrow \infty$.

Remark 2.1. The existence of bounded min-maxing sequences $\left\{A_{n}\right\}$ and $\left\{A_{n}^{k}\right\}$ in Proposition 2.1 and Proposition 2.2 respectively is not explicitly stated in the Theorems in 13 and 18 , but it hides in the proofs there.

Remark 2.2. We denote by $\operatorname{Ind}\left(u_{\lambda}\right)$ the Morse index of critical point $u_{\lambda}$ of $I_{\lambda}$. If $I_{\lambda}$ satisfies the $(P S)_{c_{\lambda}}$ condition along the min-maxing sequence $\left\{A_{n}\right\}$ in Proposition [2.1, then by Theorem 2.1 in [15] we know that there exists a critical point $u_{\lambda}$ of $I_{\lambda}$ at the critical level $c_{\lambda}$ with the Morse index $\operatorname{Ind}\left(u_{\lambda}\right) \leq 1$. If $I_{\lambda}$ satisfies the $(P S)_{c_{k}(\lambda)}$ condition along the min-maxing sequence $\left\{A_{n}^{k}\right\}$ in Proposition 2.2, then by Corollary 10.2 in [9] there exists a critical point $u_{k}(\lambda)$ of $I_{\lambda}$ at the critical level $c_{k}(\lambda)$ with the Morse index $\operatorname{Ind}\left(u_{k}(\lambda)\right) \leq k$.

Finally, we state the Liouville-type theorems. Let $\Omega=\mathbb{R}^{N}$ or $\Pi=\{x=$ $\left.\left(x_{1}, \ldots, x_{N}\right) \in \mathbb{R}^{N}: x_{N}>0\right\}$. The boundary of $\Omega$ is understood to be empty if $\Omega=\mathbb{R}^{N}$.

Proposition 2.3. Suppose that $Q$ is a function satisfying $0<\mu \leq Q \leq C$, where $\mu$ and $C$ are constants. Let $u$ be $a C_{l o c}^{2, \alpha}$-bounded solution with finite Morse index of

$$
-\Delta u=Q(x)|u|^{p-1} u \quad \text { in } \quad \Omega, \quad u=0 \quad \text { on } \partial \Omega,
$$

where $1<p<\frac{N+2}{N-2}$ if $N \geq 3$ and $p \in(1, \infty)$ if $N=2$. Then both $\|\nabla u\|_{L^{2}(\Omega)}$ and $\|u\|_{L^{p+1}(\Omega)}$ are finite. 
The Morse index of solutions of (2.1) is defined as the maximum dimension of the negative space corresponding to the spectral decomposition of the operator $-\left(\Delta+p Q u^{p-1}\right)$.

The proof of Proposition 2.3 is essentially contained in [8]. For the reader's convenience, we sketch the proof.

Take $\phi_{r, R} \in C_{c}^{\infty}\left(\mathbb{R}^{N}\right)$ such that $\phi_{r, R}=1$ over $B_{R} / B_{2 r}, \phi_{r, R}=0$ over $B_{r} \cup B_{2 R}^{c}$, and $\left|\nabla \phi_{r, R}(x)\right| \leq \frac{C}{R}, \forall x \in B_{R}^{c}$, where $R>2 r$.

Define

$$
\Phi^{\prime}(u) v=\int_{\mathbb{R}^{N}} \nabla u \nabla v d x-\int_{\mathbb{R}^{N}} Q(x)|u|^{p-1} u v d x, \quad \forall v \in C_{c}^{\infty}\left(\mathbb{R}^{N}\right),
$$

and then

$$
\Phi^{\prime \prime}(u) \psi^{2}=\int_{\mathbb{R}^{N}}|\nabla \psi|^{2} d x-p \int_{\mathbb{R}^{N}} Q(x)|u|^{p-1} \psi^{2} d x, \quad \forall \psi \in C_{c}^{\infty}\left(\mathbb{R}^{N}\right) .
$$

Lemma 2.1. Let $u$ be a solution of problem (2.1) with finite Morse index. Then there exists $r_{o}>0$ such that $\Phi^{\prime \prime}(u)\left(\phi_{r_{o}, R} u\right)^{2} \geq 0, \forall R>2 r_{o}$.

Proof. Suppose that the assertion is not true. Then for $r_{1}>0$, there exists $R_{1}>2 r_{1}$ such that $\Phi^{\prime \prime}(u)\left(\phi_{r_{1}, R_{1}} u\right)^{2}<0$ and for $r_{2}>2 R_{1}$, we may find $R_{2}>2 r_{2}$ such that $\Phi^{\prime \prime}(u)\left(\phi_{r_{2}, R_{2}} u\right)^{2}<0$. Then the supports of $\phi_{r_{1}, R_{1}} u$ and $\phi_{r_{2}, R_{2}} u$ are disjoint. So the Morse index of $u$ is larger than or equal to 2. Iterating the argument, we may get a contradiction since the Morse index of $u$ is supposed to be finite. The lemma is proved.

Proof of Proposition 2.3. By Lemma 2.1, there exists an $r_{o}>0$ such that $\Phi^{\prime \prime}(u)\left(\phi_{r_{o}, R} u\right)^{2} \geq 0, \forall R>2 r_{o}$. That is,

$$
\begin{aligned}
& \int_{\mathbb{R}^{N}}\left[|\nabla u|^{2} \phi_{r_{o}, R}^{2}+\left|\nabla \phi_{r_{o}, R}\right|^{2} u^{2}+2 u \phi_{r_{o}, R} \nabla u \nabla \phi_{r_{o}, R}\right] d x \\
& \geq p \int_{\mathbb{R}^{N}} Q(x)|u|^{p+1} \phi_{r_{o}, R}^{2} d x .
\end{aligned}
$$

Multiplying the equation by $u \phi_{r_{o}, R}^{2}$ we obtain

$$
\int_{\mathbb{R}^{N}}\left[|\nabla u|^{2} \phi_{r_{o}, R}^{2}+2 u \phi_{r_{o}, R} \nabla u \nabla \phi_{r_{o}, R}\right] d x=\int_{\mathbb{R}^{N}} Q(x)|u|^{p+1} \phi_{r_{o}, R}^{2} d x .
$$

From (2.2) and (2.3) it follows that

$$
\int_{\mathbb{R}^{N}} Q(x)|u|^{p+1} \phi_{r_{o}, R}^{2} d x \leq \frac{1}{p-1} \int_{\mathbb{R}^{N}}\left|\nabla \phi_{r_{o}, R}\right|^{2} u^{2} d x .
$$

Estimating the right side of (2.4), using the values of $\phi_{r_{o}, R}$, we get

$$
\int_{B_{R}}|u|^{p+1} d x \leq C\left(1+\frac{1}{R^{2}} \int_{B_{2 R}} u^{2} d x\right) .
$$

If $N=2$, the assertion is immediately proved from (2.5), since $u$ is bounded. If $N \geq 3$, and we assume that $\int_{\mathbb{R}^{N}}|u|^{p+1} d x$ is not finite, we obtain

$$
\int_{B_{R}}|u|^{p+1} d x \leq \frac{C}{R^{2}} \int_{B_{2 R}} u^{2} d x
$$

for large $R$. 
Using Hölder's inequality we get

$$
\int_{B_{2 R}}|u|^{2} d x \leq C\left(\int_{B_{2 R}}|u|^{p+1} d x\right)^{\frac{2}{p+1}} R^{N \frac{p-1}{p+1}},
$$

which replaced in (2.6) gives

$$
\int_{B_{R}}|u|^{p+1} d x \leq C R^{-2+N \frac{p-1}{p+1}}\left(\int_{B_{2 R}}|u|^{p+1} d x\right)^{\frac{2}{p+1}},
$$

where $C$ does not depend on $R$. After iterating (2.8), we may obtain a contradiction.

In the same way, we have

Lemma 2.2. Let $u$ be a nonnegative solution of problem (2.1) with finite Morse index. Then there exists $r_{o}>0$ such that for $R>2 r_{o}$ we have

$$
R \int_{\partial B_{R}}\left(|\nabla u|^{2}+Q(x)|u|^{p+1}\right) d S \leq C R^{N\left(\frac{p-1}{p+1}\right)-2}\left(\int_{\mathbb{R}^{N}}|u|^{p+1} d x\right)^{\frac{2}{p+1}} .
$$

\section{The PROOF OF THE MAIN RESUlts}

Let us consider the parametrized problem

$$
-\Delta u=\lambda f(x, u) \text { in } \Omega ; u=0 \text { on } \partial \Omega, \lambda \in[1,2] .
$$

Lemma 3.1. Suppose (f1)-(f4). (i) If $f(x, t) \geq 0$ for $t \geq 0$, then for almost every $\lambda \in[1,2]$, problem (3.1) possesses at least one positive $C^{2}$-solution with finite Morse index; (ii) if $f(x, t)$ is odd in $t$, then problem (3.1) possesses infinitely many solutions with finite Morse index.

Proof. (i). Since $f(x, 0)=0$ for every $x \in \Omega$, and also using the maximum principle, we may assume, without loss of generality, that $f(x, u)=f(x, 0)$ for all $x \in \Omega$ and $u \leq 0$ to find a positive solution. Let $J_{\lambda}$ be the functional associated to problem (3.1):

$$
J_{\lambda}(u)=\frac{1}{2} \int_{\Omega}|\nabla u|^{2} d x-\lambda \int_{\Omega} F(x, u) d x,
$$

where $F(x, t):=\int_{0}^{t} f(x, s) d s$. It is well known that $J_{\lambda}: H_{0}^{1}(\Omega) \rightarrow R$ is a $C^{1}$ functional. By assumptions (f1), (f2), it follows that there exist positive constants $\rho>0, \alpha>0$ such that $J_{\lambda}(u) \geq \alpha$ if $\|u\|=\rho$. On the other hand, let $\lambda_{1}$ be the first eigenvalue of the operator $-\Delta$ with zero Dirichlet condition and $\phi_{1}>0$ be the corresponding eigenfunction. Using (f4) then we have $J_{\lambda}\left(t \phi_{1}\right)<0$ for $t>0$ large. Therefore, by Proposition 2.1 for almost every $\lambda \in[1,2]$ there exists a bounded min-maxing sequence $\left\{A_{n}\right\}$ of the critical level $c_{\lambda}$ along which $J_{\lambda}$ satisfies the Palais-Smale condition. There also exists a bounded $(P S)_{c_{\lambda}}$-sequence $\left\{u_{n}\right\}$ of $J_{\lambda}$ near $A_{n}$ which in turn possesses a convergent subsequence. So for almost every $\lambda \in[1,2]$, problem (3.1) has a positive solution $u_{\lambda}$. Thus, Remark 2.2 or Theorem 2.10 of [15] implies that the Morse index ind $\left(u_{\lambda}\right)$ of $u_{\lambda}$ is less than or equal to 1 .

(ii). The proof of the existence part is the same as Lemma 3.1 in [18]. According to Remark [2.2, there exists a critical point $u_{k}(\lambda)$ of $J_{\lambda}$ corresponding to $c_{k}(\lambda)$ with the Morse index $\operatorname{Ind}\left(u_{k}(\lambda)\right) \leq k$. 
Taking $\lambda_{n} \rightarrow 1$, correspondingly, we have solutions $u_{n}:=u_{\lambda_{n}}$ and $u_{n}^{k}:=u^{k}\left(\lambda_{n}\right)$ in Lemma 3.1 with respect to $f \geq 0$ if $t \geq 0$ and $f$ is odd in $t$. The proof of Theorem 1.1 will be completed if we may prove that $\left\{u_{n}\right\}$ and $\left\{u_{n}^{k}\right\}$ are uniformly bounded in $n$ in the $L^{\infty}$-norm. Because this yields that $\left\{u_{n}\right\}$ and $\left\{u_{n}^{k}\right\}$ are uniformly bounded in $n$ in $H_{0}^{1}(\Omega)$, so it follows in a standard way that there are subsequences converging strongly in $H_{0}^{1}(\Omega)$ to solutions of (1.1). As in 18, we may deduce that if $u_{n}^{k} \rightarrow u^{k}$, $u^{k}$ are different. In the rest of this paper, we will establish uniform $L^{\infty}$-bounds for $\left\{u_{n}\right\}$ and $\left\{u_{n}^{k}\right\}$.

Let $g$ be a smooth function. We consider the problem

$$
-\Delta u=g\left(x_{o}+a x, b u\right) \text { in } \Omega, u=0 \text { on } \partial \Omega,
$$

where $a, b$ are positive constants.

We have a Pohozaev's identity for (3.3).

Lemma 3.2. Let $u$ be a solution of (3.3). Then, for any ball $B_{R}(0) \subset \Omega$ we have

$$
\begin{gathered}
b^{-1} N \int_{B_{R}} G\left(x_{o}+a x, b u\right) d x+\frac{1}{2} \int_{\partial B_{R}} R|\nabla u|^{2} d S \\
+a b^{-1} \int_{B_{R}}\left\langle x, \nabla_{x} G\left(x_{o}+a x, b u\right)\right\rangle d x \\
=\frac{N-2}{2} \int_{B_{R}}|\nabla u|^{2} d x+\int_{\partial B_{R}} R\left|\frac{\partial u}{\partial n}\right|^{2} d S+b^{-1} N \int_{\partial B_{R}} R G\left(x_{o}+a x, b u\right) d S,
\end{gathered}
$$

where $G(x, u)=\int_{0}^{u} g(x, s) d s$.

Proposition 3.1. Suppose $u_{n}$ is a solution of (3.1) with finite Morse index. Then there exists a constant $C>0$ independent of $n$ such that

$$
\left\|u_{n}\right\|_{\infty} \leq C
$$

Proof. We only consider the case $N \geq 3$. The proof is similar for $N=2$.

We argue by contradiction. Suppose that there does not exist such a constant $C$. So we should have $\left\|u_{n}\right\|_{\infty} \rightarrow \infty$ as $n \rightarrow \infty$.

We use a blow-up argument as follows.

Let $M_{n}=\max _{\bar{\Omega}}\left|u_{n}(x)\right|$ and let $x_{n} \in \Omega$ be a maximum point of $u_{n}(x)$. We define $\phi(t)=\bar{h}(t) t^{p-1}+t^{q-1}, \alpha(t)=t \phi(t)$. Let

$$
\tilde{u}_{n}(x)=M_{n}^{-1} u_{n}\left(x_{n}+\phi^{-\frac{1}{2}}\left(M_{n}\right) x\right), x \in \Omega_{n}:=\phi^{\frac{1}{2}}\left(M_{n}\right)\left(\bar{\Omega}-x_{n}\right),
$$

which satisfies

$-\Delta \tilde{u}_{n}=M_{n}^{-1} \phi^{-1}\left(M_{n}\right) \lambda_{n} f\left(x_{n}+\phi^{-\frac{1}{2}}\left(M_{n}\right) x, M_{n} \tilde{u}_{n}\right) \quad$ in $\quad \Omega_{n}, \quad \tilde{u}_{n}=0 \quad$ on $\quad \partial \Omega_{n}$ and $\left|\tilde{u}_{n}\right| \leq 1$ in $\bar{\Omega}_{n},\left|\tilde{u}_{n}(0)\right|=1$.

We may assume $x_{n} \rightarrow x_{0} \in \bar{\Omega}$. There are two cases: $x_{0} \in \Omega$ and $x_{0} \in \partial \Omega$.

Case 1. $x_{0} \in \Omega$.

Given $R>0$ there is an $n_{0} \in \mathbb{N}$ such that $\bar{B}_{2 R}(0) \subset \Omega_{n}$, for all $n \geq n_{0}$. By the interior $L^{p}$-estimates, we have for all $\gamma>1$ :

$\left\|\tilde{u}_{n}\right\|_{W^{2, \gamma}\left(B_{R}\right)} \leq C\left\{\left\|\alpha^{-1}\left(M_{n}\right) \lambda_{n} f\left(x_{n}+\phi^{-\frac{1}{2}}\left(M_{n}\right) x, M_{n} \tilde{u}_{n}\right)\right\|_{L^{\gamma}\left(B_{2 R}\right)}+\left\|\tilde{u}_{n}\right\|_{L^{\gamma}\left(B_{2 R}\right)}\right\}$.

By assumptions (f1)-(f4) we have

$$
\left.\mid \alpha^{-1}\left(M_{n}\right) \lambda_{n} f\left(x_{n}+, \phi^{-\frac{1}{2}}\left(M_{n}\right) x, M_{n} \tilde{u}_{n}\right)\right) \mid \leq C
$$

for $n$ large. Therefore

$$
\left\|\tilde{u}_{n}\right\|_{W^{2, \gamma}\left(B_{R}\right)} \leq C \quad \text { uniformly in } n .
$$


Choosing $\gamma>N$, we obtain that $\left\{\tilde{u}_{n}\right\}$ is uniformly bounded in $C^{1, \beta}\left(\bar{B}_{R}\right), 0<$ $\beta<1$. By the interior Schauder estimates one has

$$
\left.\left\|\tilde{u}_{n}\right\|_{2, \beta, B_{\frac{1}{2} R}} \leq C\left\{\| \alpha^{-1}\left(M_{n}\right) \lambda_{n} f\left(x_{n}+, \phi^{-\frac{1}{2}}\left(M_{n}\right) x, M_{n} \tilde{u}_{n}\right)\right)\left\|_{\beta, B_{R}}+\right\| \tilde{u}_{n} \|_{0, B_{R}}\right\} .
$$

Next we claim that

$$
\left.\| \alpha^{-1}\left(M_{n}\right) \lambda_{n} f\left(x_{n}+, \phi^{-\frac{1}{2}}\left(M_{n}\right) x, M_{n} \tilde{u}_{n}\right)\right) \|_{\beta, B_{R}} \leq C .
$$

To do that we write

$$
\begin{aligned}
& f\left(x_{n}+\phi^{-\frac{1}{2}}\left(M_{n}\right) x, M_{n} \tilde{u}_{n}(x)\right)-f\left(x_{n}+\phi^{-\frac{1}{2}}\left(M_{n}\right) y, M_{n} \tilde{u}_{n}(y)\right) \\
= & {\left[f\left(x_{n}+\phi^{-\frac{1}{2}}\left(M_{n}\right) x, M_{n} \tilde{u}_{n}(x)\right)-f\left(x_{n}+\phi^{-\frac{1}{2}}\left(M_{n}\right) x, M_{n} \tilde{u}_{n}(y)\right)\right] } \\
+ & {\left[f\left(x_{n}+\phi^{-\frac{1}{2}}\left(M_{n}\right) x, M_{n} \tilde{u}_{n}(y)\right)-f\left(x_{n}+\phi^{-\frac{1}{2}}\left(M_{n}\right) y, M_{n} \tilde{u}_{n}(y)\right)\right]:=I_{1}+I_{2} . }
\end{aligned}
$$

We then estimate $I_{1}$ by (f1), (f2) and (f4):

$$
\begin{aligned}
&\left|I_{1}\right| \leq \mid \frac{\partial f}{\partial t}\left(x_{n}+\right.\left.\phi^{-\frac{1}{2}}\left(M_{n}\right) x, M_{n} z_{n}\right)\left|M_{n}\right| \tilde{u}_{n}(x)-\tilde{u}_{n}(y) \mid \\
& \leq C\left(\mid h_{t}^{\prime}\left(x_{n}+\right.\right.\left.\phi^{-\frac{1}{2}}\left(M_{n}\right) x, M_{n} z_{n}\right) M_{n} z_{n} \\
& \quad+\left.p h\left(x_{n}+\phi^{-\frac{1}{2}}\left(M_{n}\right) x, M_{n} z_{n}\right)|| M_{n} z_{n}\right|^{p-1} \\
&\left.\quad+M_{n}^{q-1}+1\right) M_{n}\left|\tilde{u}_{n}(x)-\tilde{u}_{n}(y)\right| \\
& \leq C\left(h\left(x_{n}+\phi^{-\frac{1}{2}}\left(M_{n}\right) x, M_{n} z_{n}\right) M_{n}^{p-1}+M_{n}^{q-1}+1\right) M_{n}\left|\tilde{u}_{n}(x)-\tilde{u}_{n}(y)\right| \\
& \leq C \alpha\left(M_{n}\right)|x-y|^{\beta} .
\end{aligned}
$$

We use property (f2) of the function $f$ to get

$\left|I_{2}\right| \leq \mid \nabla_{x} f\left(x_{n}+\phi^{-\frac{1}{2}}\left(M_{n}\right) x, M_{n} \tilde{u}_{n}(x)\left|\phi^{-\frac{1}{2}}\left(M_{n}\right)\right| x-y\left|\leq C \phi^{-\frac{1}{2}}\left(M_{n}\right) \alpha\left(M_{n}\right)\right| x-y \mid\right.$.

Finally using (3.10) - (3.11) we obtain

$$
\alpha\left(M_{n}\right)\left|I_{1}+I_{2}\right| \leq C|x-y|^{\beta},
$$

which proves (3.8).

It follows then that

$$
\left\|\tilde{u}_{n}\right\|_{2, \beta, B_{\frac{1}{2} R}} \leq C \text { uniformly in } n .
$$

Using the Ascoli-Arzelà Theorem, (3.8) and (3.12), we obtain a subsequence of $\tilde{u}_{n}$, still denoted by $\tilde{u}_{n}$, such that

$$
\tilde{u}_{n} \rightarrow u \quad \text { in } \quad C^{2, \beta^{\prime}}\left(B_{\frac{1}{2} R}\right),
$$

and

$$
\alpha^{-1}\left(M_{n}\right) \lambda_{n} f\left(x_{n}+\phi^{-\frac{1}{2}}\left(M_{n}\right) x, M_{n} \tilde{u}_{n}(x)\right) \rightarrow A(x) \quad \text { in } \quad C^{0, \beta^{\prime}}\left(B_{\frac{1}{2} R}\right),
$$

where $0<\beta^{\prime}<\beta<1$, as $n \rightarrow \infty$. Let $\omega:=\{x: u(x) \neq 0\}$. Obviously, $\omega \neq \emptyset$. We claim that

$$
A(x) u(x) \geq \nu|u(x)|^{p+1}, \quad \forall x \in \omega .
$$

In fact, for any $x_{0} \in \omega, u\left(x_{0}\right)>0$ and there exists a constant $\delta=\delta\left(u\left(x_{0}\right)\right)>0$ such that $\tilde{u}_{n}\left(x_{0}\right) \geq \frac{1}{2} \delta$ for $n$ sufficiently large. Therefore $u_{n}\left(x_{0}\right) \rightarrow \infty$ as $n \rightarrow \infty$. 
By (f3) we obtain

$$
\begin{aligned}
A\left(x_{0}\right) u\left(x_{0}\right) & =\lim _{n \rightarrow \infty} \alpha^{-1}\left(M_{n}\right) f\left(x_{n}+\phi^{-\frac{1}{2}}\left(M_{n}\right) x_{0}, M_{n} \tilde{u}_{n}\left(x_{0}\right)\right) \tilde{u}_{n}\left(x_{0}\right) \\
& \geq \lim _{n \rightarrow \infty} C \alpha^{-1}\left(M_{n}\right)\left[\underline{h}\left(M_{n} \tilde{u}_{n}\left(x_{0}\right) M_{n}^{p}+M_{n}^{q}\right]\left|\tilde{u}_{n}\left(x_{0}\right)\right|^{p+1}\right. \\
& \geq \nu\left|u\left(x_{0}\right)\right|^{p+1} .
\end{aligned}
$$

Hence

$$
\nu|u(x)|^{p+1} \leq A(x) u(x) \leq C|u(x)|^{p+1}, \quad \forall x \in \omega .
$$

Let $Q(x)$ be a function such that $Q(x)=$ a positive constant if $x \notin \omega ; Q(x)=$ $\frac{A(x) u(x)}{|u|^{p+1}(x)}$ if $x \in \omega$. Then $A(x)=Q(x)|u|^{p-1} u$ and there exist positive constants $\sigma$ and $\gamma$ such that $\sigma \leq Q(x) \leq \gamma, \forall x \in B_{\frac{1}{2} R}$.

Passing to the limit in (3.5) and using (3.13) and (3.14), we see that $u$ satisfies

$$
-\Delta u=Q(x)|u|^{p-1} u \quad \text { in } \quad B_{\frac{1}{2} R} .
$$

By a diagonal process, this gives

$$
-\Delta u=Q(x)|u|^{p-1} u \quad \text { in } \quad R^{N} .
$$

On the other hand, if $x \in \omega$, by (f3) and L'Hospital's rule we have

$$
\begin{aligned}
Q(x)|u|^{p-1} u(x) & =\lim _{n \rightarrow \infty} \frac{\lambda_{n} f\left(x_{n}+\phi^{-\frac{1}{2}}\left(M_{n}\right) x, M_{n} \tilde{u}_{n}(x)\right)}{\alpha\left(M_{n}\right)} \\
=\lim _{n \rightarrow \infty} & \left\{\frac{\tilde{u}_{n} \lambda_{n} \frac{\partial}{\partial t} f\left(x_{n}+\phi^{-\frac{1}{2}}\left(M_{n}\right) x, M_{n} \tilde{u}_{n}(x)\right)}{\alpha^{\prime}\left(M_{n}\right)}\right. \\
& \left.-\frac{\frac{1}{2} \lambda_{n} \nabla_{x} f\left(x_{n}+\phi^{-\frac{1}{2}}\left(M_{n}\right) x, M_{n} \tilde{u}_{n}(x)\right) \phi^{-\frac{3}{2}}\left(M_{n}\right) \phi^{\prime}\left(M_{n}\right) x}{\alpha^{\prime}\left(M_{n}\right)}\right\}
\end{aligned}
$$

as $n \rightarrow \infty$. By (f2) for $x \in B_{\frac{1}{2} R}$,

$\left|\frac{1}{2} \phi^{-\frac{3}{2}}\left(M_{n}\right) \phi^{\prime}\left(M_{n}\right) \alpha^{\prime-1}\left(M_{n}\right) \nabla_{x} f\left(x_{n}+\phi^{-\frac{1}{2}}\left(M_{n}\right) x, M_{n} \tilde{u}_{n}\right) x\right| \leq C(R) \phi^{-\frac{1}{2}}\left(M_{n}\right) \rightarrow 0$

as $n \rightarrow \infty$. Since $\frac{\bar{h}^{\prime}\left(M_{n}\right) M_{n}}{\bar{h}\left(M_{n}\right)} \rightarrow-\sigma, 0 \leq \sigma<p, \frac{\alpha\left(M_{n}\right)}{M_{n} \alpha^{\prime}\left(M_{n}\right)} \rightarrow \frac{1}{p-\sigma}$. Therefore, we have

$$
\left[\alpha^{\prime}\left(M_{n}\right)\right]^{-1} \lambda_{n} \frac{\partial}{\partial t} f\left(x_{n}+\phi^{-1}\left(M_{n}\right) x, M_{n} \tilde{u}_{n}\right) \rightarrow(p-\sigma) Q(x)|u(x)|^{p-1} \quad \text { in } \quad B_{\frac{1}{2} R} .
$$

By the diagonal process, one knows that (3.18) holds also in $\mathbb{R}^{N}$ and it converges uniformly on compact sets of $\mathbb{R}^{N}$ as $n \rightarrow \infty$. Denote by $\operatorname{Ind}(p-\sigma, u)$ the Morse index of $u$ with respect to the operator $-\Delta-(p-\sigma) Q(x)|u|^{p-1}$. The uniform convergence of $u_{n}$ to $u$ on compact sets implies that the Morse index $\operatorname{Ind}(p-\sigma, u)$ of $u$ is finite (see 2] or Lemma 6 of [16]), and then $\operatorname{Ind}(p, u)$ is finite. Thus Proposition 2.1 yields that $\|u\|_{L^{p+1}\left(R^{N}\right)}$ and $\|\nabla u\|_{L^{p+1}\left(R^{N}\right)}$ are finite. We claim that $u \equiv 0$. This is a contradiction because $|u(0)|=1$. In fact, applying Lemma 3.2 to the equation 
(3.5) in the ball $B_{R}(0)$ for $R>0$ fixed we obtain

$$
\begin{gathered}
\left(M_{n} \alpha\left(M_{n}\right)\right)^{-1} N \int_{B_{R}} \lambda_{n} F\left(x_{n}+\phi^{-\frac{1}{2}}\left(M_{n}\right) x, M_{n} \tilde{u}_{n}\right) d x+\frac{1}{2} \int_{\partial B_{R}} R\left|\nabla \tilde{u}_{n}\right|^{2} d S \\
+\left(M_{n} \alpha^{3}\left(M_{n}\right)\right)^{-\frac{1}{2}} \int_{B_{R}}\left\langle x, \lambda_{n} \nabla_{x} F\left(x_{n}+\phi^{-\frac{1}{2}}\left(M_{n}\right) x, M_{n} \tilde{u}_{n}\right)\right\rangle d x \\
=\frac{N-2}{2} \int_{B_{R}}\left|\nabla \tilde{u}_{n}\right|^{2} d x+\int_{\partial B_{R}} R\left|\frac{\partial \tilde{u}_{n}}{\partial n}\right|^{2} d S \\
\quad+\left(M_{n} \alpha\left(M_{n}\right)\right)^{-1} N \int_{\partial B_{R}} R \lambda_{n} F\left(x_{n}+\phi^{-\frac{1}{2}}\left(M_{n}\right) x, M_{n} \tilde{u}_{n}\right) d S,
\end{gathered}
$$

where $F(x, t)$ is the primitive of $f(x, t)$. By (f2) we estimate

$$
\left|\left\langle x, \nabla_{x} F\left(x_{n}+\phi^{-\frac{1}{2}} x, M_{n} \tilde{u}_{n}\right)\right\rangle\right| \leq C R \alpha\left(M_{n}\right) .
$$

Therefore

$$
\left(M_{n} \alpha^{3}\left(M_{n}\right)\right)^{-\frac{1}{2}}\left|\int_{B_{R}}\left\langle x, \nabla_{x} F\left(x_{n}+\phi^{-\frac{1}{2}}\left(M_{n}\right) x, M_{n} \tilde{u}_{n}\right)\right\rangle d x\right| \leq C R\left(M_{n} \alpha\left(M_{n}\right)\right)^{-\frac{1}{2}},
$$

which tends to zero as $n \rightarrow \infty$. Using a similar argument that leads to (3.8) we can prove that the $C^{0, \alpha}$-norm of

$$
\left(M_{n} \alpha\left(M_{n}\right)\right)^{-1} F\left(x_{n}+\phi^{-\frac{1}{2}}\left(M_{n}\right) x, M_{n} \tilde{u}_{n}\right)
$$

in $B_{R}$ is uniformly bounded. Then its limit as $n \rightarrow \infty$ exists. Using (f3) and L'Hospital's rule again as in (3.17) we obtain

$$
\left(M_{n} \alpha\left(M_{n}\right)\right)^{-1} \lambda_{n} F\left(x_{n}+\phi^{-\frac{1}{2}} x, M_{n} \tilde{u}_{n}\right) \rightarrow \frac{1}{p+1-\sigma} Q(x)|u|^{p+1}(x)
$$

uniformly in $B_{R}$ as $n \rightarrow \infty$. Therefore, a use of Lebesgue's dominated convergence theorem for both the volume and the surface integrals gives

$$
\begin{aligned}
\lim _{\mathrm{n} \rightarrow \infty}\left(M_{n} \alpha\left(M_{n}\right)\right)^{-1} \int_{B_{R}} \lambda_{n} F\left(x_{n}+\phi^{-\frac{1}{2}}\left(M_{n}\right) x, M_{n} \tilde{u}_{n}\right) d x \\
\quad=\frac{1}{p+1-\sigma} \int_{B_{R}} Q(x)|u|^{p+1} d x
\end{aligned}
$$

and

$$
\begin{aligned}
\lim _{\mathrm{n} \rightarrow \infty}\left(M_{n} \alpha\left(M_{n}\right)\right)^{-1} \int_{\partial B_{R}} \lambda_{n} F\left(x_{n}+\phi^{-\frac{1}{2}}\left(M_{n}\right) x, M_{n} \tilde{u}_{n}\right) d S \\
=\frac{1}{p+1-\sigma} \int_{\partial B_{R}} Q(x)|u|^{p+1} d S .
\end{aligned}
$$

Letting $n \rightarrow \infty$ in (3.19) we obtain

$$
\begin{gathered}
\frac{N}{p+1-\sigma} \int_{B_{R}} Q(x)|u|^{p+1} d x+\frac{1}{2} \int_{\partial B_{R}} R|\nabla u|^{2} d S \\
=\frac{N-2}{2} \int_{B_{R}}|\nabla u|^{2} d x+\int_{\partial B_{R}} R\left|\frac{\partial u}{\partial n}\right|^{2} d S \\
\quad+\frac{N}{p+1-\sigma} \int_{\partial B_{R}} R Q(x)|u|^{p+1} d S .
\end{gathered}
$$

By Lemma 2.2 there exists $R \geq 2 r_{o}$ such that

$$
R \int_{\partial B_{R}}\left(|\nabla u|^{2}+Q(x)|u|^{p+1}\right) d S \leq C R^{N\left(\frac{p-1}{p+1}\right)-2}\left(\int_{\mathbb{R}^{N}}|u|^{p+1} d x\right)^{\frac{2}{p+1}} .
$$


Since $N\left(\frac{p-1}{p+1}\right)-2<0$, this implies

$$
\lim _{R \rightarrow \infty} R \int_{\partial B_{R}}\left(|\nabla u|^{2}+Q(x)|u|^{p+1}\right) d S=0 .
$$

Taking the limit $R \rightarrow \infty$ in $(3.22)$ one has

$$
\frac{2 N}{N-2} \frac{1}{p+1-\sigma} \int_{\mathbb{R}^{N}} Q(x)|u|^{p+1} d x=\int_{\mathbb{R}^{N}}|\nabla u|^{2} d x .
$$

Since $u$ is a solution of (3.16), this implies $u \equiv 0$. This completes the proof.

Case 2. $x_{0} \in \partial \Omega$.

Two cases may occur: either $d\left(x_{n}, \partial \Omega\right) \phi^{-\frac{1}{2}}\left(M_{n}\right) \rightarrow+\infty$ or $d\left(x_{n}, \partial \Omega\right) \phi^{-\frac{1}{2}}\left(M_{n}\right)$ $\rightarrow L \geq 0$ as $n \rightarrow \infty$.

If $d\left(x_{n}, \partial \Omega\right) \phi^{-\frac{1}{2}}\left(M_{n}\right) \rightarrow+\infty$ as $n \rightarrow \infty$, then $B_{n} \subset \Omega_{n}$ for $n$ large. We may obtain a contradiction in this case as Case 1 .

If $d\left(x_{n}, \partial \Omega\right) \phi^{-\frac{1}{2}}\left(M_{n}\right) \rightarrow L \geq 0$ as $n \rightarrow \infty$, by the blow-up argument we obtain a solution $u$ of

$$
-\Delta u=Q(x)|u|^{p-1} u \quad \text { in } \Pi, \quad u=0 \quad \text { on } \quad \partial \Pi
$$

with $|u| \leq 1$ in $\Pi,|u(0)|=1$ and the Morse index $\operatorname{Ind}(Q, u)$ being finite, where $\Pi=\left\{x_{1}>L\right\}$. We may deduce as Case 1 that $u \equiv 0$. This is a contradiction since $|u(0)|=1$.

\section{ACKNOWLEDGMENTS}

The work was supported by the Natural Sciences Foundation of China, No. 10271118 and the National Key Program for Basic Research of China.

\section{REFERENCES}

[1] A. Ambrosetti and P. H. Rabinowitz, Dual variational methods in critical point theory and applications, J. Funct. Anal. 14 (1973), 349 - 381. MR 51:6412

[2] A. Bahri and P. L. Lions, Solutions of superlinear elliptic equations and their Morse indices, Comm. Pure Appl. Math. 45 (1992), 1205 - 1215. MR 93m:35077]

[3] H. Berestycki, I. Capuzzo-Dolcetta, and L. Nirenberg, Superlinear indefinite elliptic problems and nonlinear Liouville theorems, Topol. Methods in Nonlinear Anal. 4 (1994), 59 - 78. MR 96d:35041

[4] H. Brézis and R. E. L. Turner, On a class of superlinear elliptic problems, Comm. Partial Differential Equations 2 (1977), 601 - 614. MR 58:23044

[5] K. C. Chang, Infinite-dimensional Morse theory and multiple solution problems, Birkhäuser, Boston, 1993. MR 94e:58023

[6] D. Costa and C. Magalhães, A variational approach to subquadratic perturbations of elliptic systems, J. Differential Equations 111 (1994), 103 - 122. MR 95f:35082

[7] D. G. de Figueiredo, P.-L. Lions, and R. Nussbaum, A priori estimates and existence of positive solutions of semilinear elliptic equations, J. Math. Pures et Appl. 61 (1982), 41 - 63. MR 83h:35039

[8] D. G. de Figueiredo and Jianfu Yang, On a semilinear elliptic problem without (PS) condition, J. Differential Equations 187 (2003), 412 - 428.

[9] N. Ghoussoub, Duality and perturbation methods in critical point theory, Cambridge University Press, Cambridge, 1993. MR 95a:58021

[10] B. Gidas and J. Spruck, A priori bounds for positive solutions of nonlinear elliptic equations, Comm. Partial Differential Equations 6 (1981), 883 - 901. MR 82h:35033

[11] B. Gidas and J. Spruck, Global and local behavior of positive solutions of nonlinear elliptic equations, Comm. Pure Appl. Math. 34 (1981), 525 - 598. MR 83f:35045

[12] H. Hofer, A geometric description of the neighbourhood of a critical point given by the mountain-pass theorem, J. London Math. Soc. 31 (1985), 566 - 570. MR 87e:58041 
[13] L. Jeanjean, On the existence of bounded Palais-Smale sequences and application to a Landesman-Lazer-type problem set on $\mathbb{R}^{N}$, Proc. Royal Soc. Edinburgh Sect. A 129 (1999), 789 - 809. MR 2001c:35034

[14] A. Harrabi, S. Rebhi, and A. Selmi, Solutions of superlinear elliptic equations and their Morse indices, I, Duke Math. J. 94 (1998), 141 - 157. MR 99i:35037

[15] M. Ramos and L. Sanchez, Homotopical linking and Morse index estimates in min-max theorems, Manuscripta Math. 87 (1995), 269 - 284. MR 96f:58031

[16] M. Ramos, S. Terracini, and C. Troestler, Superlinear indefinite elliptic problems and Pohozaev type identities, J. Funct. Anal. 159 (1998), 596 - 628. MR 2000h:35053

[17] M. Struwe, Variational methods, second edition, Springer-Verlag, Berlin, 1996. MR 98f:49002

[18] W. Zou, Variant fountain theorems and their applications, Manuscripta Math. 104 (2001), 343 - 358. MR 2002c:35081

Wuhan Institute of Physics and Mathematics, Chinese Academy of Sciences, P.O. Box 71010, Wuhan 430071, Peoples Republic of China

E-mail address: jfyang@wipm.ac.cn 